\title{
Wide-field spectroscopy of A1689 and A1835 with VIMOS: first results
}

\section{Oliver Czoske}

IAEF, Universität Bonn, Auf dem Hügel 71, 53121 Bonn, Germany

\begin{abstract}
We are using VIMOS to conduct a wide-field spectroscopic survey covering fields of $0.5 \times 0.5 \mathrm{deg}^{2}$ around the X-ray luminous clusters of galaxies Abell $1689(z=0.185)$ and Abell $1835(z=0.25)$. Here we describe the observations and first results on the redshift distribution of subsamples of cluster galaxies to $R \simeq 22$ for which we at present have obtained secure redshifts. These subsamples constitute $\sim 40 \%$ of the total spectroscopic sample and contain 525 and 630 cluster members in Abell 1689 and Abell 1835, respectively, placing them amongst the largest redshift samples available for any cluster of galaxies.
\end{abstract}

\section{Introduction}

Imaging surveys of the infall regions of clusters of galaxies which use photometric redshift techniques to isolate cluster galaxies have made strong progress in recent years (e. g. Kodama et al. 2001; Gray et al. 2003). While photometric surveys have the advantage of being complete down to well below $M^{*}$, only spectroscopic surveys can add a third dimension, velocity, to the galaxy distribution in and around clusters. The largest wide-field spectroscopic samples at present exist for near-by clusters, in particular as obtained by the CAIRNS project (Rines et al. 2003). An example for a previous wide-field redshift survey at higher redshift is described by Czoske et al. (2001, 2002), who present a catalogue of redshifts for 300 cluster members with $V \lesssim 22$ in Cl0024+1654 at $z=$ 0.395. In that case, it was only the redshift information extending to large cluster-centric distances which revealed the complex structure of what appeared in other observations to be a relaxed rich cluster.

The recent advent of high-multiplex spectrographs on 8-10 meter class telescopes has made it possible to obtain large numbers of high-quality spectra of galaxies and around clusters of galaxies in a short amount of time. The data described by Czoske et al. (2001) were obtained over the course of four years. Samples larger by a factor of $2 \ldots 3$ can now be obtained in $\sim 10$ hours of observation time. Here I present the first results from a spectroscopic survey of the two X-ray luminous clusters Abell 1689 and Abell 1835 at redshifts $z=0.185$ and $z=0.25$ respectively. We use the VIsible imaging Multi-Object Spectrograph (VIMOS) on VLT UT3/Melipal.

\section{Observations}

The field of view of VIMOS available for spectroscopy consists of four quadrants of $\sim 7 \times 7 \operatorname{arcmin}^{2}$, the separation between the quadrants is $\sim 2 \operatorname{arcmin}$. Using the LR-Blue grism, one can place $\sim 100 \ldots 150$ slits per quadrant. The resulting spectra cover the wavelength range $3700 \ldots 6700 \AA$ with a resolution $R \simeq 200$.

We use as the basis for object selection panoramic multi-colour images obtained with the CFH12k camera on CFHT (Czoske 2002), covering $40 \times 30 \operatorname{arcmin}^{2}$ in BRI for A1689 
and VRI for A1835. The input catalogue has been cleaned of stars (Bardeau et al. 2004, in preparation).

We attempted to cover the entire CFH12k field of view by using 10 VIMOS pointings for each cluster. Due to technical problems with VIMOS only 8 and 9 masks were observed in service mode for A1689 and A1835, respectively. Four of these masks did not fully meet the observational requirements although the spectra are at least partly usable.

In the observed masks, slits were placed on 3373/3542 objects in A1689 and A1835 respectively. The sample was cut at $R=23$, where objects fainter than $R=22$ were allowed to appear on several masks to provide higher signal-to-noise for these faint objects. For each mask three exposures were obtained for a total integration time of 54 minutes. The data were reduced using the VIPGI pipeline (Scodeggio et al., in preparation $\dagger$ ). The system subtracts a bias frame, automatically localises the $2 \mathrm{D}$ spectra on the chip and determines their curvature. After wavelength calibration from HeNeAr standard lamp observations the 1D spectra were optimally extracted.

Redshift determination involves an identification step using cross correlation with a set of templates and a subsequent interactive check of the correctness of the redshift identification, and a measurement step using again a correlation with the allowed redshift range constrained to a narrow interval around the correct identification. At the time of writing secure redshifts have been determined for 1312/1427 objects in the two clusters. All further results presented in this contribution will be based on these subsets which reach $R \sim 22$ and constitute $\sim 40 \%$ of the total samples.

\section{Redshift space structure}

\subsection{Abell 1689}

Abell 1689 is one of the richest $(R=4)$ clusters in the Abell catalogue and shows a complex structure in its inner parts, including a large number of strongly gravitationally lensed arcs and arclets. Its X-ray luminosity and temperature are $L_{\mathrm{X}}=$ $5.2 \times 10^{44} h^{-2} \mathrm{erg} \mathrm{s}^{-1}$ (Ebeling et al. 1996) and $T_{\mathrm{X}}=9.3 \mathrm{keV}$ (Andersson \& Madejski 2004), respectively. Based on 96 redshifts in the cluster centre, Girardi et al. (1997) divide the redshift distribution into three distinct subgroups.

The left panel of Fig. 1 shows the redshift distribution for galaxies near the mean cluster redshift of Abell 1689 as derived from our VIMOS data. We include redshifts from the literature and previous observations of the cluster centre using $4 \mathrm{~m}$ class telescopes. We define cluster membership by the redshift range $0.169<z<0.202$ and find 525 cluster members in the subsample.

The distribution on large scales is very regular, with clearly defined "caustics" whose amplitude decreases strongly with clustercentric distance. This is also reflected in the velocity dispersion profile which decreases from $\simeq 2100 \mathrm{~km} \mathrm{~s}^{-1}$ in the central bins to $\simeq 1200 \mathrm{~km} \mathrm{~s}^{-1}$ for the outer bins. The redshift histogram in conjunction with the redshiftdistance scatter plot hints at the presence of a distinct subgroup in the cluster center at a clustercentric velocity of $\sim 3000 \mathrm{~km} \mathrm{~s}^{-1}$ which might artificially enhance the central velocity dispersion.

In order to probe for the presence of substructure in Abell 1689 we apply the test described by Dressler \& Shectman (1988). This test computes for each galaxy the statistic $\delta \sim\left[\left(\bar{v}_{\text {local }}-\bar{v}\right)^{2}+\left(\sigma_{\text {local }}-\sigma\right)^{2}\right]$ to compare the local velocity distribution based on the 10 nearest neighbours to the global distribution. The left panel in Fig. 2 shows the result

$\dagger$ http://cosmos.mi.iasf.cnr.it/marcos/vipgi/vipgi.html 

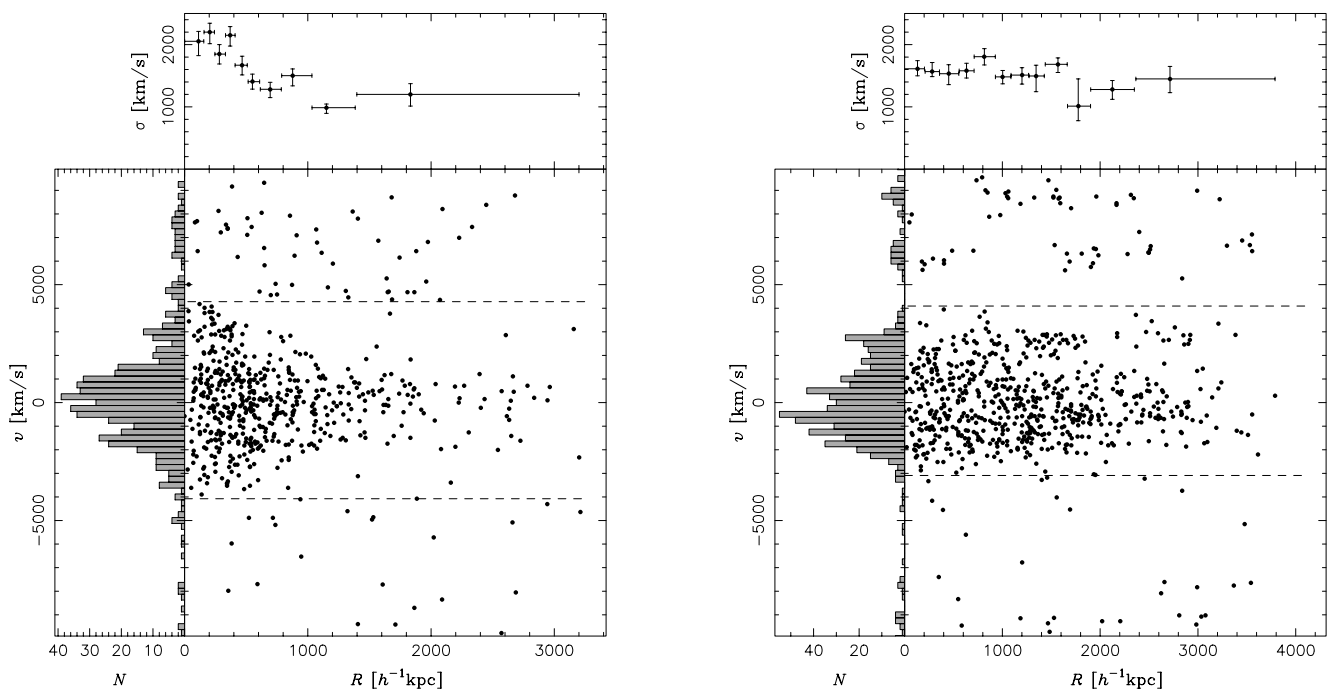

Figure 1. The redshift distribution for Abell 1689 (left) and Abell 1835 (right). Redshifts are given as cosmologically corrected velocities relative to the mean cluster redshifts. The velocity dispersion profiles in the top panels were computed in bins containing 50 galaxies each within the cluster limits marked in the central panel.
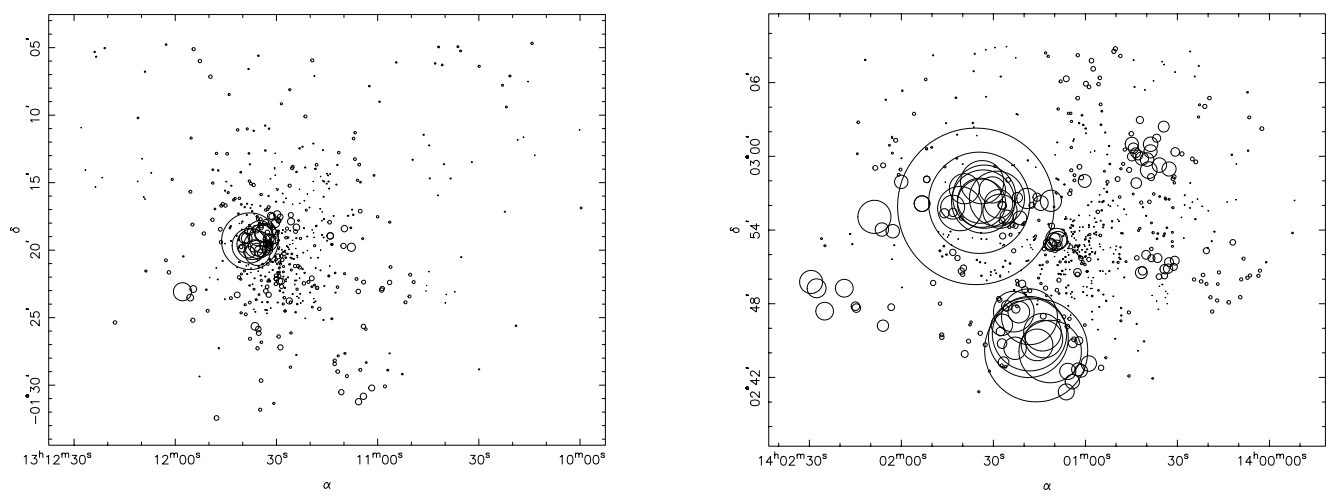

Figure 2. Dressler-Shectman plots for Abell 1689 (left) and Abell 1835 (right). The circles have radii proportional to $\exp (\delta)$, where $\delta$ is the Dressler-Shectman statistic (see text for details).

for Abell 1689. The only apparent distinct group of galaxies lies at $\sim 2^{\prime}$ to the northeast of the cluster center. The location of this group, which has been seen in previous redshift samples, corresponds to a group of bright galaxies in images of the cluster. The distribution of redshifts of these galaxies is skewed towards higher redshifts: $51 \%$ of them lie at $z>0.192$, whereas only $13 \%$ of all cluster members lie at this redshift. The distribution of all galaxies with $z>0.192$ covers a much larger field but seems concentrated on the location identified by the Dressler-Shectman test. Andersson \& Madejski (2004) use X-ray data obtained with XMM/Newton to derive a redshift map for the emitting intra-cluster gas and find evidence for a higher redshift in their eastern quadrant, roughly coinciding with our substructure. 


\subsection{Abell 1835}

Abell 1835, with $9.6 \times 10^{44} h^{-2} \mathrm{erg} \mathrm{s}^{-1}$, is the most luminous cluster in the ROSAT Brightest Cluster Sample (Ebeling et al. 1998). Temperature measurements with ASCA and XMM-Newton find a comparatively modest global temperature of $\sim 7.5 \mathrm{keV}$ (Ota 2001; Majerowicz et al. 2002) with a cool core of about $4 \mathrm{keV}$. The surface brightness distribution is nearly circular to a radius of $\sim 0.8 h^{-1} \mathrm{Mpc}$. From observations of the central parts of Abell 1835 it therefore appears that this is a very massive and relaxed cluster.

The redshift distribution around Abell 1835 is shown in the right hand panel of Fig. 1. Defining cluster membership by $0.24<z<0.27$, we find 630 cluster members. The distribution of Abell 1835 seems more extended than the distribution of Abell 1689, although a detailed investigation of the completeness of the samples is necessary to assess the validity of this statement. The velocity dispersion profile (VDP) of the cluster galaxies in Abell 1835 is flat at $\sim 1500 \mathrm{~km} \mathrm{~s}^{-1}$. From the histogram and the redshiftdistance plot it is clear, however, that the VDP at large radii includes a contribution from a seemingly separate band of redshifts at $\sim 3000 \mathrm{~km} \mathrm{~s}^{-1}$ and might thus be overestimated at $R \gtrsim 1 h^{-1} \mathrm{Mpc}$.

The result of the Dressler-Shectman test on Abell 1835 is shown in the right hand panel of Fig. 2. It is immediately apparent that the infall region is much more structured than the one of Abell 1689, indicating that these clusters inhabit rather different environments. The Dressler-Shectman test provides evidence for at least two distinct structures in the outskirts of Abell 1835, one $\sim 1.5 h^{-1} \mathrm{Mpc}$ to the east of the cluster center, the other $\sim 1.4 h^{-1} \mathrm{Mpc}$ to the south-east. The eastern structure is centred on a compact group of eight galaxies, all with $z>0.26$, significantly higher than the mean cluster redshift. The southern structure is centred around a bright elliptical galaxy for which unfortunately no redshift is available. The mean redshift of the galaxies in this group is 0.245 , somewhat lower than the cluster redshift.

\section{Conclusions}

The project described in this contribution is still at an early stage. So far, secure redshifts have been obtained for only $\sim 40 \%$ of the observed samples. Nevertheless, the subsamples presented here include 525 and 630 members galaxies of the clusters Abell 1689 and Abell 1835, respectively. Already, these samples are the largest available for any cluster with the exception of Coma (Rines et al. 2003), and demonstrate the power of VIMOS to provide unprecedented redshift samples for clusters of galaxies at intermediate redshifts.

We have described the redshift distribution for the two clusters out to $\gtrsim 3 h^{-1} \mathrm{Mpc}$ $\left(\gtrsim 2 r_{\text {vir }}\right.$ ). The infall regions of the two clusters are markedly different, with Abell 1835 showing evidence for strong clustering in its outskirts whereas the outskirts of Abell 1689 seems much more homogeneous. Curiously, this is at contrast to the structure of the central parts of the clusters which is complex for Abell 1689 but seems relaxed for Abell 1835. The spatially more concentrated galaxy distribution in Abell 1689 as compared to Abell 1835 agrees with results from weak lensing analyses by Clowe \& Schneider (2001, 2002), who find concentration parameters of $c=7.9$ for Abell 1689 and $c=2.9$ for Abell 1835.

The first goal of future work is to complete the redshift determination for the spectral samples and thus to extend the study of the cluster structures to fainter magnitude. After proper assessment of the completeness characteristics of the resulting galaxy catalogues 
it will be possible to provide mass profiles based on integration of the Jeans equation in the inner parts and analysis of the "caustic" structure in the outer parts, following Diaferio (1999), which can then be compared to mass profiles derived from weak lensing (Clowe \& Schneider 2001, 2002; Bardeau et al. 2004).

Further work will relate the spectral types of the galaxies to cluster-centric distance, the local galaxy density and surface mass density as determined from weak gravitational lensing.

\section{References}

Andersson, K. E. \& Madejski, G. M. 2004, astro-ph/0401604

Bardeau, S., Kneib, J.-P., Czoske, O., Richard, J., Soucail, G., Smail, I., Ebeling, H., \& Smith, G. P. 2004, in preparation

Clowe, D. \& Schneider, P. 2001, Astron. \& Astroph. , 379, 384

-. 2002, Astron. \& Astroph. , 395, 385

Czoske, O. 2002, PhD thesis, Université Toulouse III - Paul Sabatier

Czoske, O., Kneib, J.-P., Soucail, G., Bridges, T. J., Mellier, Y., \& Cuillandre, J.-C. 2001, Astron. E Astroph. , 372, 391

Czoske, O., Moore, B., Kneib, J.-P., \& Soucail, G. 2002, Astron. \& Astroph. , 386, 31

Diaferio, A. 1999, Mon. Not. Roy. Ast. Soc. , 309, 610

Dressler, A. \& Shectman, S. A. 1988, Astron. Journ. , 95, 985

Ebeling, H., Edge, A. C., Böhringer, H., Allen, S. W., Crawford, C. S., Fabian, A. C., Voges, W., \& Huchra, J. P. 1998, Mon. Not. Roy. Ast. Soc. , 301, 881

Ebeling, H., Voges, W., Böhringer, H., Edge, A. C., Huchra, J. P., \& Briel, U. G. 1996, Mon. Not. Roy. Ast. Soc. , 281, 799

Girardi, M., Fadda, D., Escalera, E., Giuricin, G., Mardirossian, F., \& Mezzetti, M. 1997, Astroph. Journ. , 490, 56

Gray, M. E., Wolf, C., Meisenheimer, K., Taylor, A., Dye, S., Borch, A., \& Kleinheinrich, M. 2003, astro-ph/0312106

Kodama, T., Smail, I., Nakata, F., Okamura, S., \& Bower, R. G. 2001, Astroph. Journ. , 562, L9

Majerowicz, S., Neumann, D. M., \& Reiprich, T. H. 2002, Astron. \&6 Astroph. , 394, 77

Ota, N. 2001, PhD thesis, University of Tokyo

Rines, K., Geller, M. J., Kurtz, M. J., \& Diaferio, A. 2003, Astron. Journ. , 126, 2152 